\title{
A Survey of Mental Health of New Patients at Traditional Chinese Medical Clinics
}

\author{
Siu Man Ng, Ted Chun Tat Fong, Xiao Lu Wang \\ Centre on Behavioral Health, The University of Hong Kong, Hong Kong, China \\ E-mail:ngsiuman@hku.hk \\ Received October 4, 2010; revised December 5, 2010; accepted December 9, 2010
}

\begin{abstract}
In Chinese society, traditional Chinese medical clinics are popular and play a significant role in primary healthcare. The current study aimed to examine the mental health of new patients at Chinese medical clinics. A cross-sectional questionnaire survey was conducted. Subjects were new patients of a Chinese medical clinic recruited through $100 \%$ consecutive sampling during a 5-week period. A response rate of $84.2 \%$ and a sample size of 367 were achieved. Samples included 111 (30.2\%) men and 256 (69.8\%) women. Mean age was 44.0 (SD = 17.1). Presenting problems were all somatic, with $68.3 \%$ lasted over 1 month and $37.1 \%$ lasted over 1 year. In the month prior to the first consultation, 26.4\%, 33.2\% and 19.3\% patients consulted other public, private and Chinese medical clinics on average 1.5, 1.9 and 3.0 times respectively. Besides, 7.4\% and 6.5\% patients self-administered Western and Chinese drugs on average 1.3 and 2.4 times respecttively. Using a cut-off of $\geq 5$ on GHQ-12, prevalence of common mental disorders was estimated at $21.0 \%$. In conclusion, findings revealed that common mental disorders were prevalent among new Chinese medicine patients although presenting problems of subjects were all somatic. Many of these presenting medical conditions were chronic, with $68.3 \%$ lasting over 1 month and 37.1\% lasting over 1 year. Over $70 \%$ patients had been seeking various forms of treatment in the month prior to first consultation. Mental health and illness behavior of Chinese medicine patients warrants further research and attention.
\end{abstract}

Keywords: Alternative Medicine, Mental Health, Primary Healthcare

\section{Introduction}

Mental disorders are common among the Chinese people [1]. Adopting a 2-stage design, that is a questionnaire screening followed by a standardized diagnostic interview, the overall lifetime prevalence of mental disorders among adults in the community was reported at $18.9 \%$ in Hong Kong [2]. If tobacco dependence and pathological gambling were included, the overall prevalence was $29.1 \%$. Since most people with mental disorders will show up in primary healthcare, although largely for somatic discomforts, primary healthcare theoretically may play a pivotal role in the community psychiatry framework [3,4].

Despite rapid modernization and socioeconomic changes, traditional Chinese medicine (TCM) remains popular among the Chinese people and is generally perceived as "better in treating chronic illnesses", "curing underlying causes" and "more gentle and having fewer side effects” [5]. Over the past decade in Hong Kong, TCM has been systematically formalized and incorporated into modern healthcare system. According to the Chinese Medicine Council of Hong Kong, there were about 5,853 registered Chinese medicine practitioners (RCMP) as at April 2009. For a city of seven million people, the figure translates into one RCMP per population of about 1,200 , suggesting that TCM clinics are playing a significant role in primary healthcare.

A key characteristic of the illness behavior of the Chinese people is a strong tendency to express concern or solicit attention through somatic discomforts (e.g. see the classic work of Kleinman \& Kleinman, 1985 [6]). With a root in Chinese culture, TCM has evolved to manage this 'somatization' tendency through a strategicholistic approach. While the somatic complaints are fully acknowledged, the higher goal is to treat both mind and body. For example, depression is generally considered as equivalent to the 'stagnation syndrome' of TCM [7]. A 
key difference, however, is that stagnation syndrome has a prominent somatic component characterized by obstruction-like discomforts at throat, chest, stomach or abdomen. An emphasis on somatic symptoms makes it socially legitimate to seek medical care. Stagnation syndrome and other 'mind/body disorders' are often examined by general Chinese medicine practitioners in the community. It is thus reasonable to expect that many Chinese people with mental disorders turn up at TCM clinics. However, systematic studies on this area are still awaited.

The objective of the current study is to investigate the incoming pathways and overall prevalence of common mental disorders of new patients of a TCM clinic of a major healthcare provider in Hong Kong. The scope of the study did not include returning patients mainly because of methodological considerations. Returning patients had big heterogeneity in terms of stage of recovery and duration of treatment at the clinic. Besides, there might be significant systemic differences between returning and defaulting patients. Access to defaulting patients for making such assessment would be difficult. From a methodological research perspective, the mental health profile of returning patients had to be investigated by a substantially more sophisticated design in future studies.

Ethical approval for this study was sought from the Joint Institutional Review Board of the University of Hong Kong and the Hospital Authority Hong Kong West Cluster, with compliance to the Declaration of Helsinki.

\section{Methods}

1) Participants: current study adopted a cross-sectional questionnaire survey design. Participants were recruited through $100 \%$ consecutive sampling of new patients of a TCM clinic during a pre-designated 5-week period. Inclusion criteria were: 1 ) being a 'new case' in accordance to the clinic's administrative definition, i.e. a patient who is seeking treatment for a certain medical condition for the first time, or presenting at a particular sub-specialty of the clinic for the first time; 2) if the patient is aged under 13 or mentally unfit to be interviewed, he/she is accompanied by a carer who can be the informant; and 3) the patient or, if applicable, his/her carer can communicate effectively in Cantonese or Putonghua. During the designated study period, 436 subjects meeting inclusion criteria were approached. A response rate of $84.2 \%$ was achieved, resulting in 367 completed questionnaires. There were 111 (30.2\%) male and 256 (69.8\%) female. Mean age was 44.0 years $(\mathrm{SD}=17.1)$.

2) Interviewers: six part-time interviewers were specifically employed for the study by the investigating in- stitution. All of them were retired nurses with around 20 to 30 years of clinical and management experience in public hospitals in Hong Kong. They were trained on administering the questionnaires by the first author, principal investigator of the study.

3) measures:

- Demographic data: These included gender, age, marital status, educational level, personal and family income, type of housing, religious beliefs and household composition.

- Incoming pathway: This section captured patients' help-seeking behaviors in the month prior to first consultation at the TCM clinic, i.e. (1) methods used to deal with the presenting health problem, and (2) frequency of using the method(s).

- Mental Health: The 12-Item General Health Questionnaire (GHQ-12) [8] is a self-administered screening instrument for identifying non-psychotic psychiatric disorders in community settings. It has been widely used in primary care settings and its measurement properties have been studied in diverse cultural settings [3]. The Chinese version has been validated and found to have satisfactory psychometric properties, with sensitivity and specificity at $78 \%$ and $77 \%$ respectively $[9,10]$. For estimation of overall prevalence of common mental disorders, current study adopted the 0-0-1-1 coding and a cut-off point of $\geq 5$ [11].

4) procedures: At any one time during the 5-week data collection period, two to three interviewers stationed at the clinic for conducting interviews following a structured questionnaire. All new patients were invited to participate in the study. Consented patients were interviewed individually in a room with privacy inside the clinic. All completed questionnaires were sent to the principal investigator directly. The clinic had no access to the collected data.

\section{Results}

1) Duration of Presenting Problems: Regarding the duration of the presenting problems, the key findings are summarized in Table 1 below.

2) Results showed a wide range in the duration of presenting problems. While $31.7 \%$ presenting problems had lasted for less than a month at first consultation, 37.8\% had lasted for over 1 year. Overall, 68.3\% presenting problems had lasted for over 1 month.

3) Treatments Used Prior to First Consultations: Participants were asked whether they had employed any methods to deal with their presenting health problems in the month prior to the first consultation. The major findings are summarized in Table 2 below. 
Table 1. Frequency counts of duration of presenting problems at first consultation.

\begin{tabular}{lcc}
\hline & f & \% \\
\hline $\mathbf{1}$ wk & 36 & 14.6 \\
$\mathbf{1 - 2}$ wks & 14 & 5.7 \\
$\mathbf{2 w k s - 1}$ mth & 28 & 11.4 \\
$\mathbf{1 - 6}$ mths & 41 & 16.7 \\
$\mathbf{6 m t h s - 1}$ yr & 34 & 13.8 \\
$\mathbf{1 - 5}$ yrs & 40 & 16.7 \\
$\mathbf{5 - 1 0}$ yrs & 18 & 7.3 \\
$>\mathbf{1 0}$ yrs & 34 & 13.8 \\
\hline
\end{tabular}

Note: $\mathrm{f}=$ frequency count.

Table 2. Treatments used on presenting problems in the month prior to first consultation.

\begin{tabular}{lccc}
\hline \multirow{2}{*}{ Methods used } & \multicolumn{2}{c}{ Patients reported use } & $\begin{array}{c}\text { Mean no. } \\
\text { of times } \\
\text { used }\end{array}$ \\
\cline { 2 - 4 } & $\mathrm{n}$ & $\mathrm{n} / \mathrm{N}(\%) \dagger$ & 1.5 \\
\hline Public clinic & 97 & $26.4 \%$ & 1.9 \\
Private clinic & 122 & $33.2 \%$ & 3.0 \\
Chinese medical clinic & 71 & $19.3 \%$ & 1.3 \\
Self-medication: western drug & 27 & $7.4 \%$ & 2.4 \\
Self-medication: Chinese medicine & 24 & $6.5 \%$ & - \\
Others & 13 & $3.5 \%$ & - \\
No action & 89 & $24.3 \%$ & - \\
\hline
\end{tabular}

$\dagger$ Note: $\mathrm{N}=367$. Since some participants had used more than one method to deal with their presenting problems, the percentages added over $100 \%$.

4) Many patients (75.7\%) had sought other treatments in the month prior to first consultation at the clinic. Using other private, public and TCM clinics was common (26.4\%, 33.2\% and 19.3\% patients respectively). These patients on average had 2.0 consultations elsewhere in the previous month. Regarding self-medication, 7.4\% and $6.5 \%$ patients self-administered Western and Chinese drugs respectively.

5) Mental Health: Mean GHQ-12 score of the sample was 2.67 (SD 2.91). If a cut-off of $\geq 5$ was adopted, the implied overall prevalence of common mental disorders among new patients of the TCM clinic was $21.0 \%$.

\section{Discussions}

The clinic handled a significant amount of chronic conditions. Sixty eight percent patients had their presenting problems which had lasted for over a month at first con- sultation. Thirty eight percent had even lasted for over a year. Most patients had sought other treatments and/or used self- medication prior to consultation. From an efficiency and effectiveness perspective, it seems necessary to explore alternative intervention modalities for supplementing the conventional one-to-one medical consultation model. In light of the promising results of the chronic illness self-management (CISM) model in western countries [12], it seems worthwhile experimenting similar programs at TCM clinics. By incorporating culturally relevant elements into CISM, such as some traditional herbal, dietary and physical health practices, the programs may be more appealing and motivational to local patients, leading to better long-term compliance.

The findings suggested that common mental disorders were common among new TCM patients, with an overall prevalence estimated at $21.0 \%$. Theoretically TCM clinics may play a role in a community psychiatry framework and help people with mental health problems [13]. To realize these potentials, however, much further work is still needed. In light of the experience in the west, substantial enhancement of Chinese medicine undergraduate syllabus and continued professional training of practicing RCMPs seems necessary. At the systemic level, it seems desirable to encourage multidisciplinary collaborations at TCM clinics so as to better addressing the multidimensional needs of patients.

\section{Conclusions}

Findings of the current study revealed that although the presenting problems of new patients were all somatic, common mental disorders were prevalent among them. A substantial proportion of the presenting problems were chronic conditions. Chinese medical clinics may potentially be a good contact point for delivering integrative mental health care and chronic disease management in the community.

\section{Acknowledgements}

The study was funded by Tung Wah Hospital, a nonprofit organization in Hong Kong.

\section{References}

[1] Q. C. Shi, J.M. Zhang, F. Z. Xu, R. P. Michael, Y. Xu, Y. L. Fu, W. Gu, X. J. Zhou, S. M. Wang, Y. Zhang and M. $\mathrm{Yu}$, "Epidemiological Survey of Mental Illnesses in the People Aged 15 and Older in Zhejiang Province, China," Zhonghua Yu Fang Yi Xue Za Zhi, Vol. 39, No. 4, pp. 229-236.

[2] C. N. Chen, J. Wong, N. Lee, M. W. Chan-Ho, J. T. F. Lau and M. Fung, "The Shatin Community Mental 
Health Survey in Hong Kong,” Archives of General Psychiatry, Vol. 50, No. 2, 1993, pp. 125-133.

[3] T. B. Ustun and N. Sartorius, "Mental Illness in General Health Care-An International Study,” John Wiley \& Sons, Chichester, 1995.

[4] D. Goldberg, "Epidemiology of Mental Disorders in Primary Care Settings,” Epidemiologic Reviews, Vol. 17, No. 1, 1995, pp. 182-190.

[5] T. P. Lam, "Strengths and Weaknesses of Traditional Chinese Medicine and Western Medicine in the Eyes of Hong Kong Chinese," The Journal of Epidemiology and Community Health, Vol. 55, No. 10, 2001, pp. 762-765. doi:10.1136/jech.55.10.762

[6] A. Kleinman and J. Kleinman, "Somatization: The Interconnections in Chinese Society Among Culture, Depressive Experiences, and the Meanings of Pain,” In: A. Kleinman and B. Good Eds., Culture and DepressionStudies in the Anthropology and Cross-Cultural Psychiatry of Affect and Disorder, University of California Press, Berkeley, 1985, pp. 429-490.

[7] S. M. Ng, C. L. W. Chan, D. Y. F. Ho, Y. Y. Wong, and R. T. H. Ho, "Stagnation as a Distinct Clinical Syndrome: Comparing 'Yu' (Stagnation) in Traditional Chinese Medicine with Depression," British Journal of Social Work, Vol. 36, No. 3, 2006, pp. 467-484. doi:10.1093/bjsw/bcl008

[8] D. Goldberg, "The Detection of Mental Illness by Ques- tionnaire,” Oxford University Press, London, 1972.

[9] M. Y. Chong and G. Wilkinson, "Validation of 30- and 12-Item Versions of the Chinese Health Questionnaire (CHQ) in Patients Admitted for General Health Screening,” Psychological Medicine, Vol. 19, No. 2, 1989, pp. 495-505. doi:10.1017/S0033291700012526

[10] D. T. Shek, "Reliability and Factorial Structure of the Chinese Version of the General Health Questionnaire," Journal of Clinical Psychology, Vol. 43, No. 6, 1987, pp. 683-691. doi:10.1002/1097-4679(198711)43:6<683::AID -JCLP2270430606>3.0.CO;2-B

[11] D. P. Goldberg and Y. Lecrubier, "Form and Frequency of Mental Disorders Across Centres,” In: T.B. Ustun and N. Sartorius Eds., Mental Illness in General Health Care: An International Study, John Wiley \& Sons (On behalf of WHO), Chichester, 1995, pp. 323-334.

[12] K. Lorig, “Self-Management in Chronic Illness,” In: S. G. Funk, Ed., Key Aspects of Preventing and Managing Chronic Illness, Springer, New York, 2001.

[13] S. M. Ng, H. M. Ng and Y. Tong, "The Role of Chinese Medical Clinics in Detecting and Treating Mental Disorders- A Social Psychiatry Perspective,” Journal of Traditional Chinese Medicine, Vol. 45(Supp.), 2004, pp. 1112. 